\title{
Valognes - Alleaume
}

$\mathrm{n}^{\circ} 3355$

Laurent Paez-Rezende, Laurence Jeanne, Caroline Duclos, Christophe Brémont, Pierre-Marie Guihard, Magali Heppe, Gaël Léon, Amélie Marie et Laurent Vipard

\section{(2) OpenEdition}

\section{Journals}

Édition électronique

URL : http://journals.openedition.org/adlfi/16799

ISSN : 2114-0502

Éditeur

Ministère de la culture

Référence électronique

Laurent Paez-Rezende, Laurence Jeanne, Caroline Duclos, Christophe Brémont, Pierre-Marie Guihard, Magali Heppe, Gaël Léon, Amélie Marie et Laurent Vipard, «Valognes - Alleaume », ADLFI. Archéologie de la France - Informations [En ligne], Basse-Normandie, mis en ligne le 26 février 2016, consulté le 03 mai 2019. URL : http://journals.openedition.org/adlfi/16799

Ce document a été généré automatiquement le 3 mai 2019.

(C) Ministère de la Culture et de la Communication, CNRS 


\title{
Valognes - Alleaume
}

$n^{\circ} 3355$

\author{
Laurent Paez-Rezende, Laurence Jeanne, Caroline Duclos, Christophe \\ Brémont, Pierre-Marie Guihard, Magali Heppe, Gaël Léon, Amélie Marie \\ et Laurent Vipard
}

Lien Atlas (MCC) :

http://atlas.patrimoines.culture.fr/atlas/trunk/index.php?

ap_theme=DOM_2.01.02\&ap_bbox=-1.514;49.448;-1.437;49.536

1 La ville antique d'Alleaume, à Valognes, est située à 1,6 km au sud-est du centre-bourg médiéval et actuel. À la différence de ce dernier, positionné sur la bordure de la petite confluence du Merderet et du ruisseau Saint-Jean, Alauna s'est implantée sur le plateau de la rive sud des sources du Merderet et, plus précisément, sur son rebord nord-ouest.

2 L'emprise urbaine, révélée par les sondages de juillet 2013, a investi le plateau entre deux petits talwegs, d'orientation globalement sud-nord, qui soulignent les limites orientales, où est implanté le théâtre antique, et occidentales, où se trouvent des carrières et des zones d'épandage. Seuls les thermes nord, fouillés entre 1989 et 1992, sont installés sur le pied de versant de la vallée du Merderet. Dans l'intervalle, entre les thermes et les aménagements urbains mis au jour, soit la bordure nord du plateau et la pente, la prospection géophysique de 2012 n'a révélé que de très faibles indices et la prospection thématique, de la même année, ne met pas en évidence d'aussi grandes concentrations de mobiliers que sur les parcelles investies en sondages.

Reconnue depuis la fin du XVII ${ }^{e}$ siècle par l'intermédiaire de ses principaux monuments, thermes et théâtre, l'agglomération antique de l'ancien village d'Alleaume, aux portes sud-est de la ville de Valognes, s'étendrait sur une surface estimée entre 20 et 35 ha. Dès l'origine des recherches, le statut de cette ville antique a été l'objet de discussions et de débats. Parfois hâtivement assimilée à l'énigmatique Crociatonum, elle est surtout adoptée par la communauté scientifique comme étant la station Alauna de la Table de Peutinger et de l'Itinéraire d'Antonin. Elle est aussi, depuis lors, au cœur des discussions sur son hypothétique fonction de chef-lieu de la Civitas des Unelles, territoire qui en est toujours dépourvu. Force est de constater, qu'en l'état, aucun document épigraphique, 
bibliographique ou archéologique, ne permet de trancher sur ces sujets. Surtout, depuis trois siècles, prospections, fouilles, sondages et études diverses n'ont toujours pas permis de définir la configuration exacte de cette agglomération, de mesurer avec précision son étendue et son organisation, de vérifier le taux d'occupation et ses variations, de compléter la connaissance de sa parure monumentale et surtout de fixer les grandes étapes chronologiques de son évolution.

4 La stratégie de sondage d'intervention, définie en concertation avec le SRA de BasseNormandie, s'est limitée à l'ouverture d'une seule tranchée dans la diagonale de chaque parcelle. Ce procédé éprouvé permet de disposer les ouvertures en décalage avec les lignes directrices du paysage actuel, et surtout avec celles du schéma urbain que la prospection géophysique avait clairement révélé en 2012.

5 Au final, c'est un bilan très positif qui émane de cette première année de sondages car, en dépit des ouvertures pour le moins exigües, des avancées significatives dans les connaissances sur la ville antique d'Alleaume ont été réalisées. Elles portent sur les trois aspects que recouvrait la problématique initiale: l'état de conservation des vestiges, l'organisation de la ville et la chronologie.

6 Au cœur de l'espace urbain, des rues et des ruelles, dont la chaussée est revêtue de petits galets à l'évidence calibrés, se croisent à angle droit et délimitent sans conteste des quartiers d'habitations; des sols en béton de chaux ou en galets, des murs en moellons calcaires ou blocs de grès, des restes de fours ou de foyers et même un égout construit en tuiles y matérialisent l'emplacement de ces édifices résidentiels. Sur la périphérie, les bâtiments et les rues se raréfient pour laisser la place aux structures d'organisation du paysage, notamment des fossés qui délimitent les parcelles cultivées ou les pâtures, reprenant les orientations des rues. Dans ces espaces, on décèle également des activités artisanales, comme la production de chaux ou de métal (forge) et des indices de boucherie, mais aussi des carrières, accompagnées par quelques bâtiments. Partout les fosses, les dépotoirs, la céramique en abondance, le matériel métallique, les ossements d'animaux ou les restes de coquillages, les morceaux d'enduits peints ou de revêtements de sols, les monnaies, etc., viennent témoigner d'une occupation intense et longue.

7 Ainsi, les huit parcelles investies ont toutes livré des vestiges, en majorité gallo-romains ; toutefois, ceux-ci ne présentent pas partout un état de conservation homogène. Ce différentiel a été cartographié et permet de distinguer quatre zones. Il s'agit là d'une image synthétique, mettant en évidence les caractères généraux de la conservation et de la densité des indices archéologiques mais qui, en même temps, dessine une organisation schématique révélant les principaux espaces de la ville, à savoir la zone urbanisée, véritablement enchâssée dans le maillage des rues, et la zone périphérique. Plus concrètement, l'intervention a révélé sur ce point un potentiel stratigraphique que, ni les fouilles programmées des thermes au tournant des années 80 et 90 , ni les sondages ponctuels de l'archéologie préventive, n'avaient pu mettre en évidence. On notera notamment, sur des points ciblés, l'existence de séquences d'occupation multiples et complexes pouvant intégrer jusqu'à cinq phases d'aménagements; ou bien encore de restructurations urbaines de plus grande ampleur, sans doute à l'échelle de tout un quartier, et qui s'entrevoient notamment dans l'abandon de certains axes de voiries que viennent coloniser de nouveaux édifices.

8 Cette cartographie des états de conservation est intéressante sur le plan patrimonial, car elle révèle les deux grands espaces de la ville qui caractérisent ce site. Il s'agit, en premier lieu, de la zone urbaine proprement dite, c'est-à-dire l'emprise située à l'intérieur du 
périmètre délimité par le réseau des voies où se concentre l'essentiel des vestiges d'habitat, de circulation, d'ostentation et de consommation. Dans ce périmètre, les vestiges sont véritablement enchâssés dans le maillage des rues dont l'organisation orthogonale, déjà révélée par la prospection géophysique de 2012, et largement complétée par cette campagne de terrain, délimite assurément des insulae où prennent place de nombreux édifices maçonnés et tout un cortège de vestiges qui leur sont associés. De plus, ces sondages auront permis de situer les limites physiques occidentale et méridionale de la ville, qui sont matérialisées par deux grands axes de voirie au-delà desquels, vers le sud comme vers l'ouest, se développe la zone dite péri-urbaine.

Il s'agit là d'une zone de contact à la structuration plus lâche ou plus ciblée, remisant les activités non admises dans le périmètre urbain mais pourtant nécessaires à son fonctionnement. Ainsi se diffusent, sur une large bande s'appuyant sur les rives externes des deux voies "d'interface ", des réseaux parcellaires aussi bien organisés que le réseau des rues, quelques habitats, des dépotoirs et surtout des traces d'activités artisanales (métallurgie, boucherie...); il ne manque que les nécropoles, absence qui pourrait bien signifier que les limites de l'urbs n'ont finalement pas été atteintes et que cette zone périphérique de l'espace urbain appartient encore à la ville.

La révision de la chronologie n'était pas le moindre des objectifs tant l'état des connaissances sur la chronologie de cette agglomération apparaissait déficiente. En effet, avant cette intervention, l'évolution de la ville était purement et simplement calquée sur la chronologie des thermes nord, fouillés par T. Lepert, que l'on peut ainsi résumer : un démarrage de l'occupation au milieu du $\mathrm{I}^{\mathrm{er}} \mathrm{s}$., suivi d'une phase d'agrandissement à la fin du $\mathrm{I}^{\mathrm{er}} \mathrm{s}$. ou au début du $\mathrm{II}^{\mathrm{e}} \mathrm{s}$., et un abandon avant la fin du $\mathrm{III}^{\mathrm{e}}$ s. Même si ces sondages n'ont pas permis de mener une étude détaillée de la céramique, les ensembles qui en sont issus ont néanmoins bénéficié d'une expertise succincte, validant les grandes lignes directrices d'une chronologie rénovée. Ainsi, des éléments attestent d'une occupation sous-jacente située vers la fin du II ${ }^{\mathrm{e}}$ s. av. J.-C. et le courant du $\mathrm{I}^{\mathrm{er}}$ s. av. J.-C. Sans lien apparent avec ce fond de la fin de l'âge du Fer, les ensembles les plus précoces indiqueraient un démarrage de l'agglomération dans la période augustéenne, soit entre la toute fin du $\mathrm{I}^{\mathrm{er}}$ s. av. J.-C. et le premier quart du $\mathrm{I}^{\mathrm{er}}$ s. apr. J.-C. Ensuite, les lots les plus nombreux et les plus volumineux, répartis dans tous les secteurs sondés, appartiennent à des phases d'occupation comprises entre le milieu du $\mathrm{I}^{\mathrm{er}} \mathrm{s}$. et la fin du $\mathrm{III}^{\mathrm{e}} \mathrm{s}$.

11 Au-delà du $\mathrm{III}^{\mathrm{e}} \mathrm{s}$., la chronologie est plus difficile à restituer. Pour le moment, les productions de l'Antiquité tardive n'ont pas encore été identifiées. Les quelques productions médiévales révèlent, d'une part, des fréquentations ponctuelles dans le haut Moyen Âge, au sens large, avant que ne se fixent, autour des XII ${ }^{\mathrm{e}}-\mathrm{XIII}{ }^{\mathrm{e}}$ s., de nouveaux habitats et notamment un édifice, sur les marges sud de la ville, qui semblent marquer la mise en place du nouveau hameau de la Victoire. Il faut également signaler des découvertes récurrentes d'éléments couvrant toute la période moderne et contemporaine, signes d'une campagne active et consécutives de la fixation de plusieurs grosses entités rurales encore en activité.

En conclusion, cette première année d'intervention est riche d'enseignements sur le potentiel de cette agglomération et dans tous les domaines de recherches. Mais l'apport le plus marquant de ce programme de recherche réside dans l'approche des faubourgs de cette cité, espace souvent détruit par des aménagements diachroniques ou bien, quand il est préservé, trop souvent délaissé par les investigations archéologiques. En 2014 et 2015, 
l'exploration continuera, de la même manière, à préciser la configuration et l'extension de ces deux espaces.

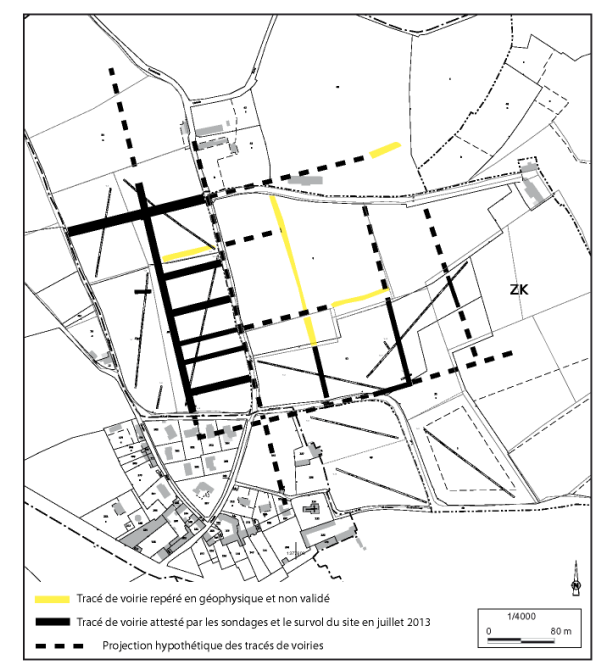

Fig.01

Schéma urbain défini par le réseau des rues

DAO : L. Paez-Rezende, Inrap

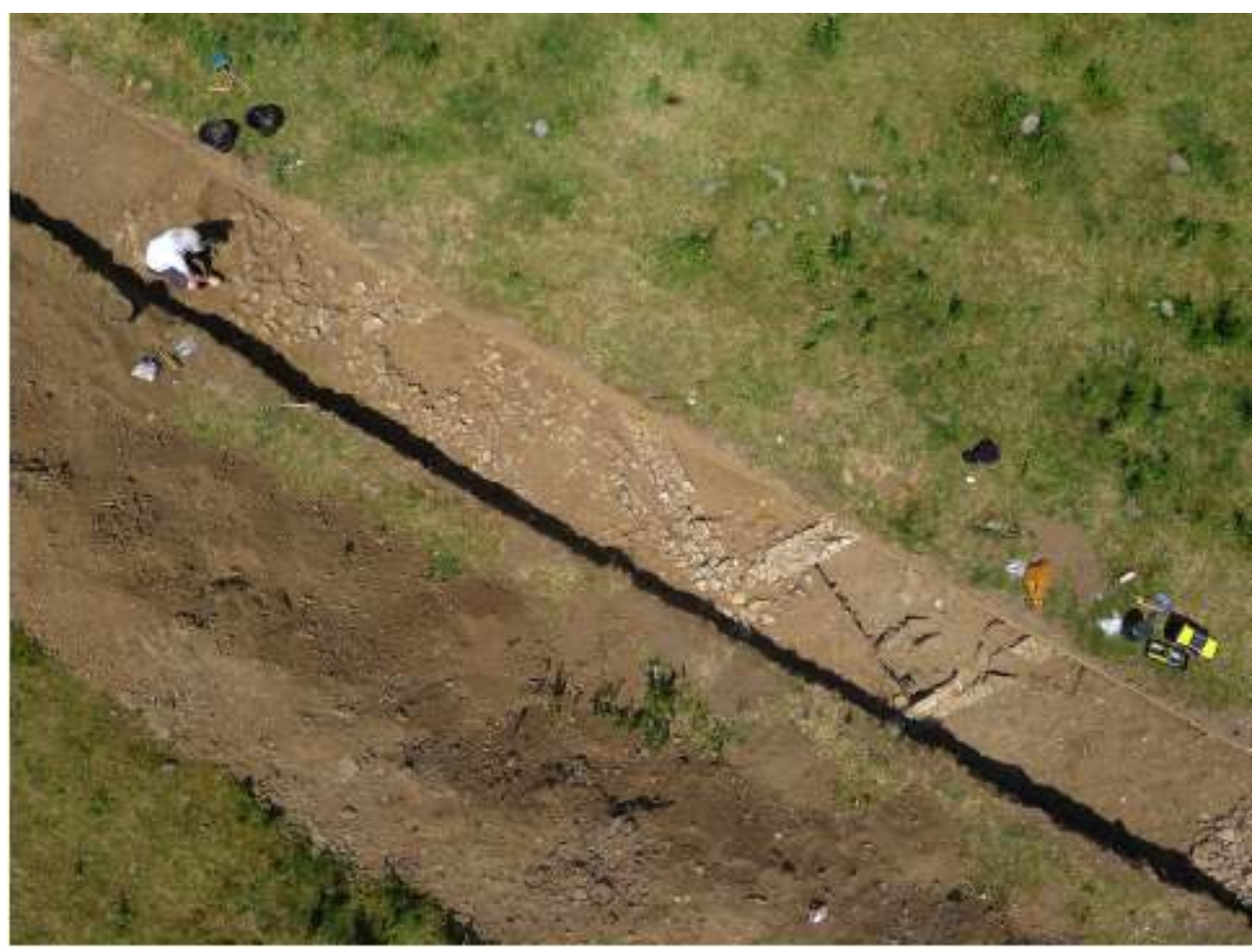


Fig. 02

Vue aérienne par cerf-volant de vestiges de maçonneries

Cliché : F. Levalet

INDEX

Index chronologique : Antiquité

Index géographique : Basse-Normandie, Manche (50), Valognes

Mots-clés : voirie, bâti, zone urbaine, faubourgs

operation Sondage (SD)

\section{AUTEURS}

LAURENT PAEZ-REZENDE

Inrap 